\title{
Co-worker Robot - "PaDY"
}

\section{Jun KINUGAWA $\dagger$, Yusuke SUGAHARA $\ddagger$, Kazuhiro KOSUGE $\dagger$}

$\dagger$ Department of Bioengineering and Robotics, Graduate School of Engineering, Tohoku University, 6-6-01 Aoba, Aramaki, Aobaku, Sendai 980-8579, JAPAN, kinugawa@irs.mech.tohoku.ac.jp, kosuge@irs.mech.toho-ku.ac.jp $\ddagger$ Tokyo Institute of Technology, 2-12-1 Ookayama, Meguroku, Tokyo 152-8552, Japan, sugahara@mech.titech.ac.jp

Abstract: We present the first developed prototype of the co-worker robot "PaDY" (i.e., intime Parts/tools Delivery to You) for automotive assembly processes and demonstrate its effectiveness in assembling vehicles. PaDY delivers the necessary part(s) and tool(s) for each task, to a worker during the assembly process, increasing efficiency, by reducing unnecessary movements by the worker; this also reduces the load of the worker and mistakes. Here we introduce the concept underlying PaDY and illustrate how PaDY can be applied to an actual assembly process based on our previous articles[3]-[6].

Keywords: Human-Robot Interaction; Partner Robot; Co-worker Robot;

\section{Introduction}

Industrial robots are developed to replace human workers involved in dirty, dangerous, and demanding tasks, or "3D" tasks. Today, however, industrial robots cannot fully replace human workers in all production stages. Actually, many complex tasks are still performed by skilled human workers.

With the rapid worldwide aging of the population, replacing human workers has become a key issues for future production systems. A realistic solution is to reduce their workload and to increase efficiency.

The development of a robot system that can cooperate with human workers to reduce their load and increase efficiency and quality of work is attracting much attention in the development of "next-generation" robots, such as, YuMi, developed by $\mathrm{ABB}$ and Baxter, developed by Rethink Robotics.

As assembly task support system in automobile production line, Toyota Motor Corporation has developed a skill-assist system for handling a heavy instrument panel module[1] and a front and rear windows installation assist system[2]. These systems were developed for assisting a worker physically. 


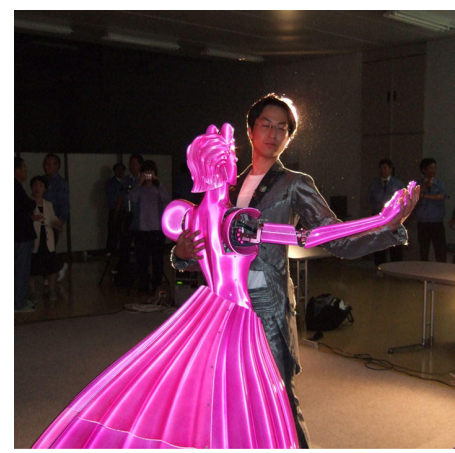

Figure 1

Dance Partner Robot "PBDR"

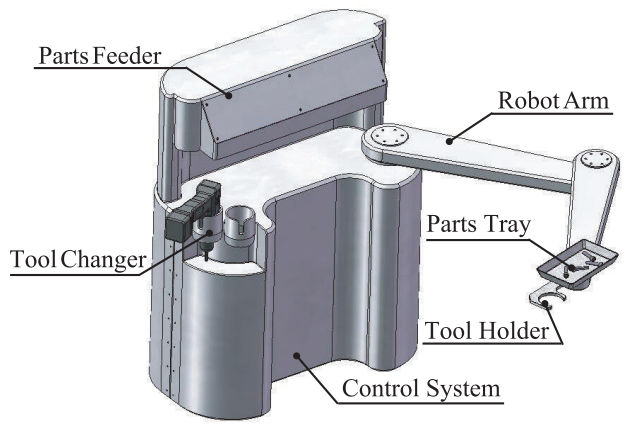

Figure 2

Concept of Co-worker Robot PaDY for Automobile Assemble Process

The co-worker robot "PaDY" was developed in our laboratory as a prototype for the assembly process in the automotive production system. In this article, we will introduce the outline of PaDY [3]-[6], which we are developing, and illustrate how they are utilized in a production system.

\section{Co-worker Robot PaDY}

\subsection{Concept}

PaDY has been developed, based on the results of our previous study on the dance partner robot "PBDR" (i.e., Partner Ballroom Dance Robot) [7](Fig. 1). PBDR is a female dance partner robot, which could perform a ballroom dance along with a human male dancer. PBDR could understand its dance partner's lead, estimate the fnext dance steps and dance in coordination with the male partner. Estimating the male dancer's lead was the key issue in developing PBDR, along with realizing the stable physical human-robot interaction between PBDR and its partner.

When we visited an automotive assembly factory in Japan, we came across an interesting scene. An assembly process comprises a certain number of tasks, which have to be completed in a predetermined order defined by the working process schedule. The workers in certain assembly processes were noted to spend a lot of time selecting and picking up the necessary parts and tools before attaching these to the vehicle being assembled, which consumes a significant amount of time within this process.

A robot that is capable of estimating the worker's next move or anticipating the next assembly task could probably provide the worker with the necessary part(s) and tool(s) as and when required. This is the concept of the co-worker robot "PaDY" (i.e., in-time Parts/tools Delivery to You). PaDY is capable of delivering the necessary parts and tools to a human worker when he/she needs them for each task in the assembly process.

PaDY is expected to reduce unnecessary actions involved in assembling parts to a vehicle, such as walking to the storage (i.e., a place where parts and tools for 


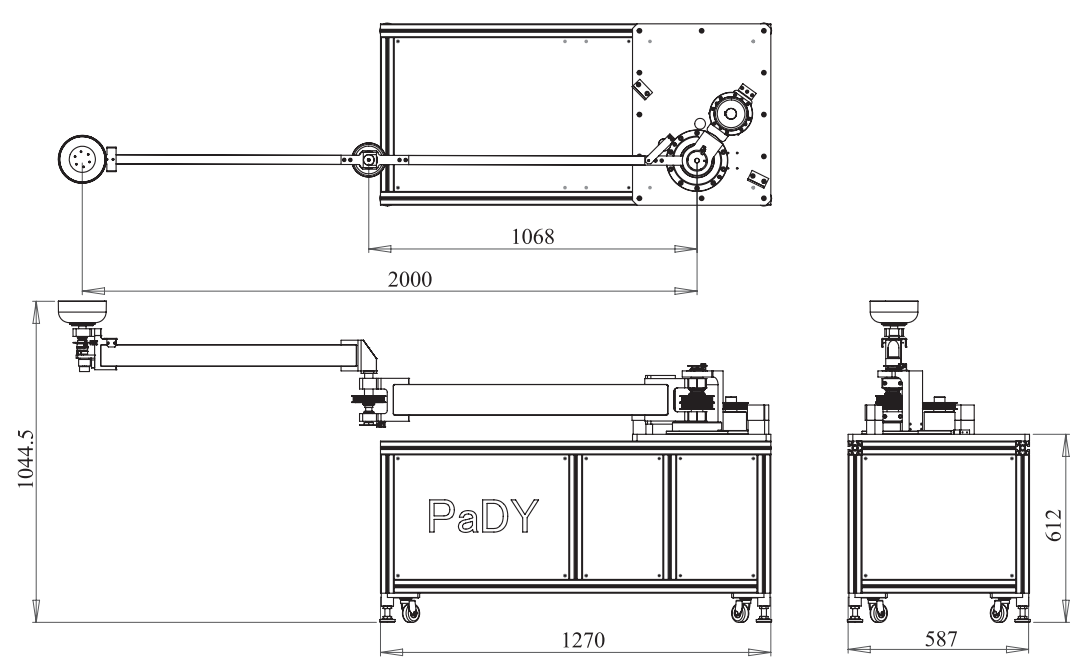

(a) Dimensions of PaDY P1

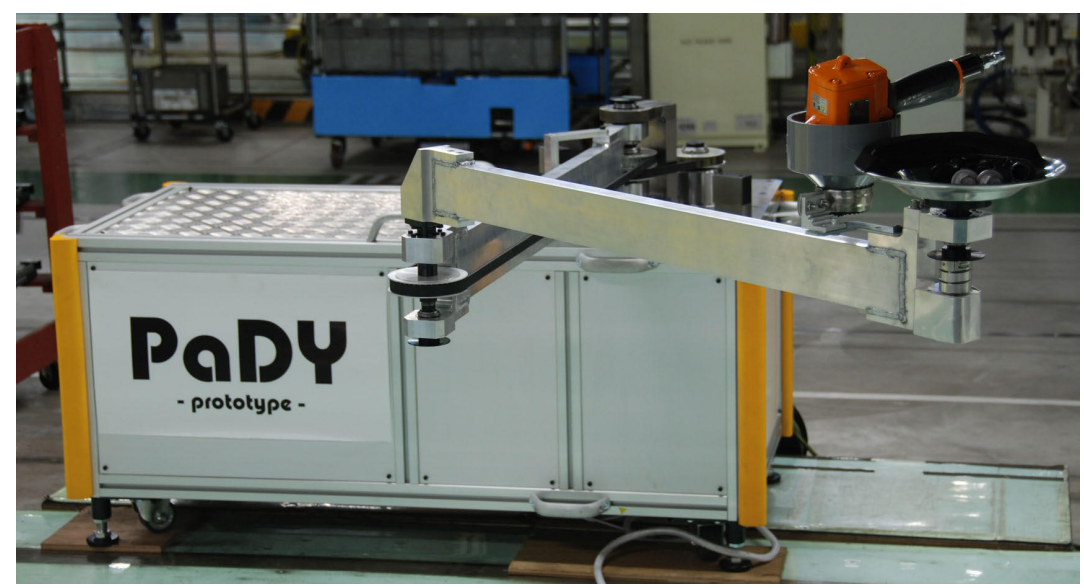

(b) Photo of PaDY P1

Figure 3

The Co-worker robot PaDY Prototype P1

the assembly process are stored), which is usually located close to the assembly line; selecting and picking up the necessary part(s) and tool(s) from the storage; and returning to the place where the assembly was being performed. Consequently, the assembly process can be conducted more efficiently using PaDY. In addition, possible mistakes, such as selection of incorrect part(s) and tool(s) for the next task, could be reduced as PaDY automatically selects the necessary part(s) and tool(s) according to the reference process of the assembly process.

\subsection{System Configuration}

PaDY consists of a manipulator system for delivering parts and tools to a human worker; a measurement system, which is used to locate the worker; part feeders, which place the necessary part(s) onto a tray attached to the end of the manipulator; 


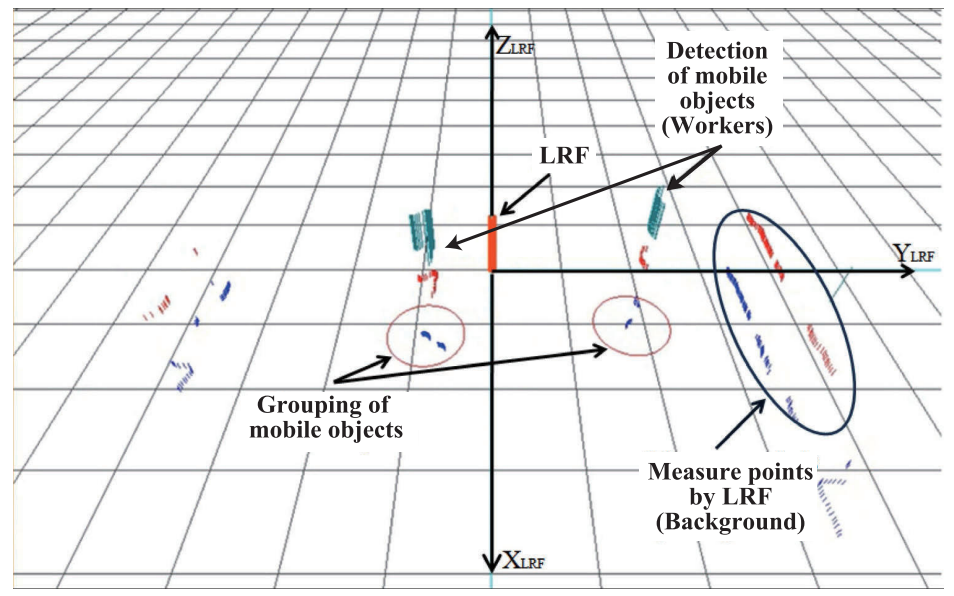

Figure 4

Worker Detection by Using Background Subtraction Technique and a Clustering Method

a tool changer for selecting the necessary tool(s); and an integrated control system.

The integrated control system includes the work progress estimation and arm motion planning systems. The work progress estimation system estimates the current state of the work process based on information from the measurement system, while referring to the working process schedule a priori. The motion planning system controls the manipulator by planning a trajectory of the arm in accordance with the work progress obtained via the work progress estimation system.

First, the worker is located using the laser range finder (LRF) system; subsequently, the integrated control system decides the appropriate delivery location and time at which the part(s) and tool(s) are required for the next task based on the history of the worker's position and the work progress model of the working process. PaDY then delivers the part(s) and tool(s) to the worker by controlling the manipulator based on the motion planned. The system architecture is shown in Fig. 2.

The co-worker robot prototype PaDY P1 is presented in Fig. 3. Note that for simplification, peripheral systems such as the motion measurement system, part feeders, and tool changers have not been included in the figures.

\subsection{Manipulator Design for PaDY}

PaDY coexists with the worker and operates within the worker's workspace. The manipulator was designed based on the following requirements:

- Wide range of motion: The manipulator needs to have a sufficient range of motion to cover the entire workspace.

- Lightweight: To reduce the impact force for unavoidable/possible collisions with the worker, the manipulator needs to be as lightweight as possible.

- Low power actuators: To ensure safety, low power actuators are incorporated. 


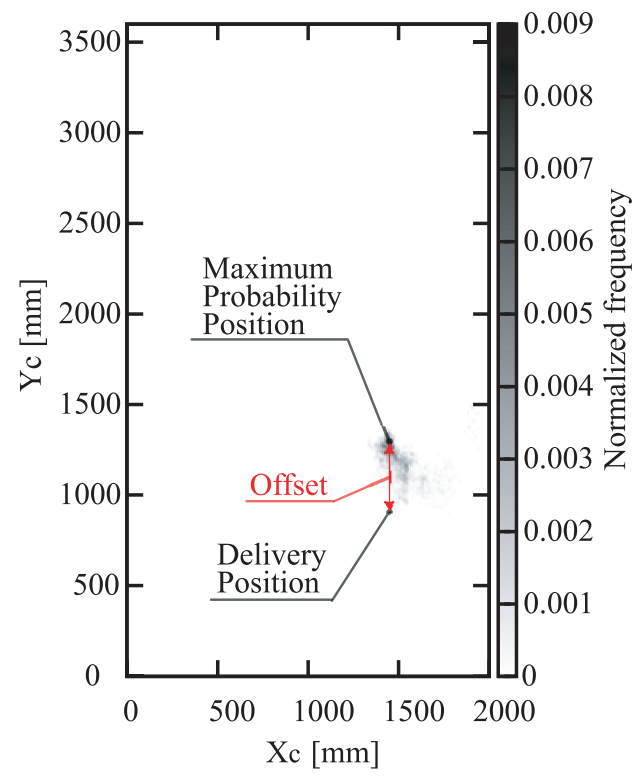

Figure 5

Delivery Position

- Load capacity: The manipulator needs to have adequate load capacity for carrying the part(s) and tool(s) required for each task of the assembly process.

We developed the Very Light Weight Wide Workspace (VLWWW) Arm to meet the requirements listed above. The VLWWW arm is driven by servomotors through mechanical torque limiters to guarantee that excessive force is not applied to its environment, including the worker in the environment.

\section{Motion Planning Based on a Statistical Approach}

\subsection{Locating the Workers}

In the prototype system, the worker is located using the LRF system. It is because the LRF system could cover wide space enough to cover the wide range workspace. Assuming that the worker is the only object that moves in the workspace being considered, the background subtraction method is used for extracting the worker from the measurement data by the LRF system. The workers in the workspace are detected by applying a clustering method to the measurement data processed by the background subtraction method[6].

In the prototype system, the nearest neighbor method[8] is used, and a set of measurement points located within a circle with a certain radius (in the following example, $500 \mathrm{~mm}$ ) relative to a point of the set are regarded to belong to the same cluster. The number of clusters obtained by this technique is regarded as the number of the workers in the same workspace. Figure 4 shows an example of identifying and locating the workers using this method. 


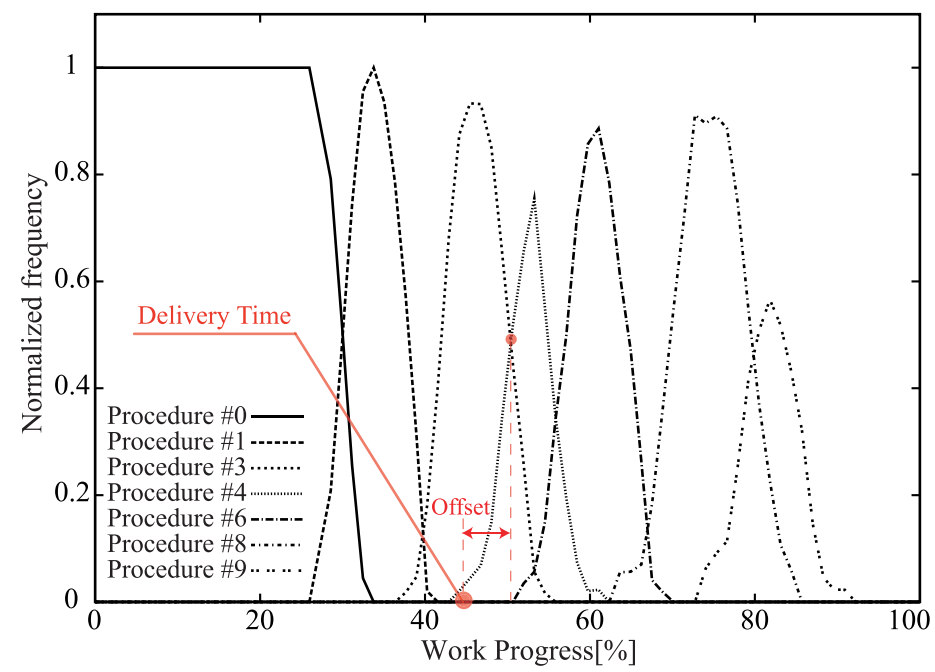

Figure 6

Delivery Time(Tasks $\# 0, \# 2, \# 5$, and $\# 7$ are not shown in this figure because these are not assembly tasks.)

\subsection{Motion Planning System}

Ordinal industrial robots are controlled using the teach-in and playback system; this system is quite useful for simple, repetitive operations. However, the teach-in and playback concept could not be applied to the co-worker robot because the human worker could not repeat the exact same motion in repetitive operations. To solve this problem, we developed the motion planning system, which generates robot motion based on the work progress model constructed by statistically processing the worker's motion data.

\subsubsection{Statistical Analysis of the Assembly Process}

To statistically analyze the assembly process and to understand how each task is performed, the worker is localized in a coordinate system attached to the body of the vehicle being assembled. The workspace described in the coordinate system attached to the body of the vehicle is divided into cells by a grid with an appropriate size.

The worker's position is periodically measured by the LRF system. Notably, the vehicle moves during any assembly process; therefore, each assembly task considered in this study was performed at a different location.

We calculate the existence probability of the worker $E_{n, i, j}$ in each cell $(i, j)$ for $n$-th task of the assembly process by dividing the frequency recorded for the cell $(i, j)$ for $n$-th task by the total number of data points recorded for all of the tasks involved in the assembly process for the cell $(i, j) . E_{n, i, j}$ shows the probability that the worker is located in this cell $(i, j)$ during the $n$-th task of the process.

The frequency $F_{n, t}$ that the worker is engaged in the $n$-th task at time $t$ from the start 


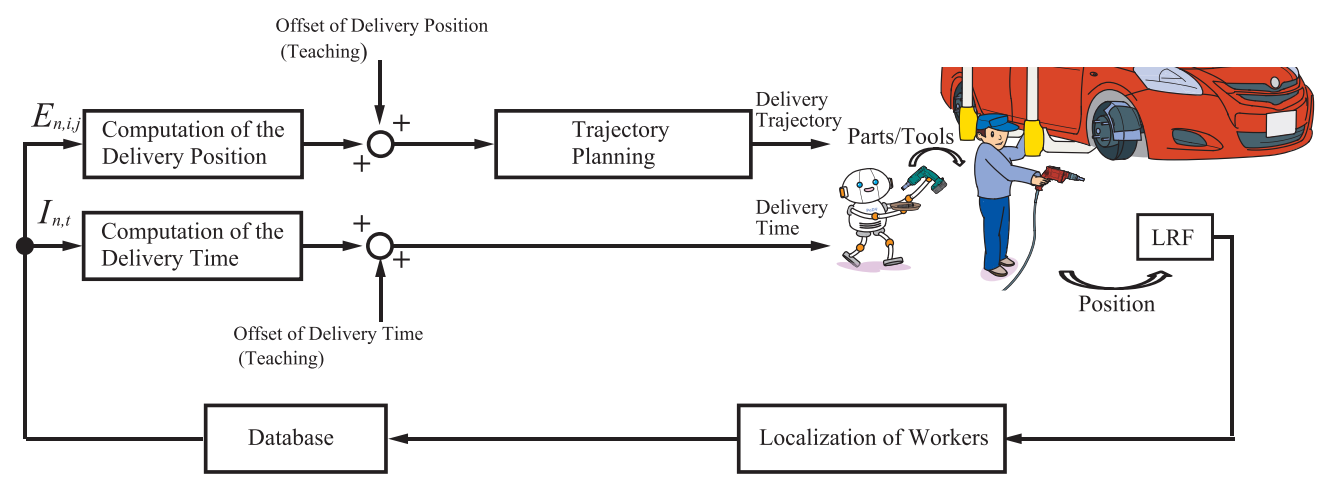

Figure 7

Control System

of each assembly process is also repeatedly recorded. The execution probability for the $n$-th task at time $t, I_{n, t}$, is computed by dividing $F_{n, t}$ with which the worker is engaged in the $n$-th task at $t$ by the total number of the recorded repetitions of the assembly process. Therefore, we could estimate which task the worker is executing at $t$ using $I_{n, t}$.

The frequency $G_{n, i, j}$ with which the worker is engaged in the $n$-th task in a particular cell $(i, j)$ is also recorded. The execution probability of the $n$-th task for a given cell $(i, j), R_{n, i, j}$, is calculated by dividing $G_{n, i, j}$ by the total number of recorded frequencies for the cell $(i, j)$ for all of the tasks and for all the repetitions of the assembly process. $R_{n, i, j}$ indicates the probability that the worker is engaged in the $n$-th task in the cell $(i, j)$.

The manipulator's motion is planned based on the probabilities defined above.

\subsubsection{Motion Planning}

In the motion planning system, the time and location at which the part(s) and tool(s) will be delivered is determined and the manipulator's trajectory is generated. The outline of the motion planning is shown below:

1. The worker's location is estimated using the data measured by the LRF system.

2. The existence probability of the worker $E_{n, i, j}$, execution probability of $n$-th task at time $t, I_{n, t}$, execution probability of $n$-th task for a given cell $(i, j)$, and $R_{n, i, j}$ are calculated using the recorded data for a certain number of repetitions of the assembly process.

3. The delivery position of the part(s) and tool(s) for the $n$-th task is determined by the existence probability $E_{n, i, j}$. That is, the cell having the highest probability is the location where the $n$-th task is executed, as each task in the assembly process is defined considering the location of work with respect to the vehicle body when the assembly process is segmented into tasks. The 


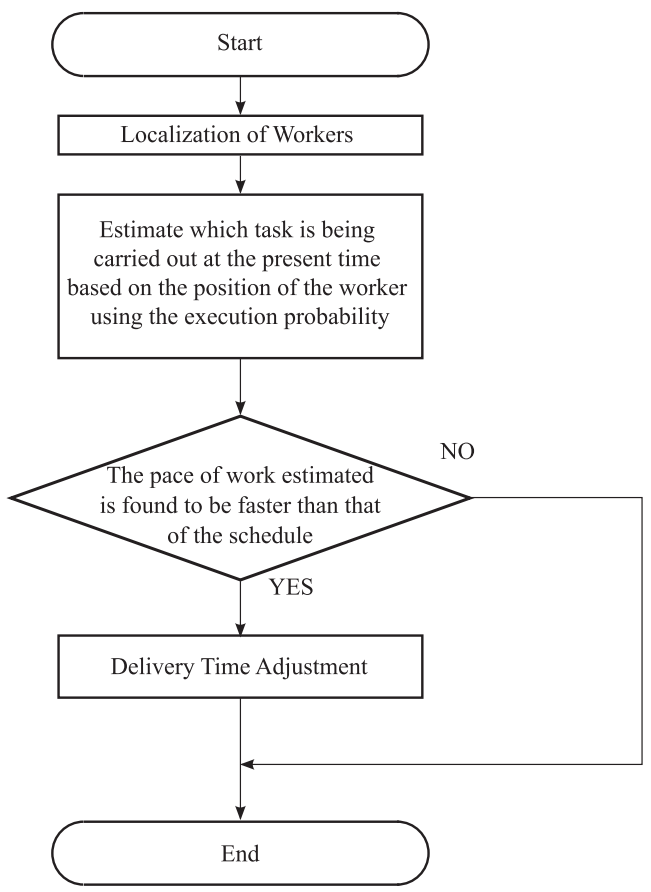

Figure 8

Delivery Time Adjustment Based on Worker's Position information

delivery position of the part(s) and tool(s) is appropriately determined for the worker based on the delivery position determined above by adding the "offset" position to the selected location (Fig. 5).

4. The delivery time of the part(s) and tool(s) of the $n$-th task is determined by the execution probability $I_{n, t}$ of the $n$-th task at time $t$. That is, the delivery time is determined based on the time when the probability of the $n$-th task exceeds the probability of the $(n-1)$-th task. The delivery time is determined by subtracting an offset time from this time (Fig. 6).

5. The trajectory of the manipulator is generated based on the delivery position and time of the part(s) and tool(s).

Note that the aforementioned planning is conducted for each task of the assembly process being considered. Figure 7 illustrates the architecture of the control system based on the motion planning system described above.

\subsubsection{Delivery Time Adjustment}

In the method stated above, the start of each task is determined under the assumption that the worker is engaged in the task, which has the highest execution probability with respect to time. If some of the tasks up to the $(n-1)$-th task are performed earlier than usual, the worker needs to wait until the part(s) and tool(s) arrive at the location for the $n$-th task, as the start of each task is determined based on the highest execution probability of tasks with respect to time. 


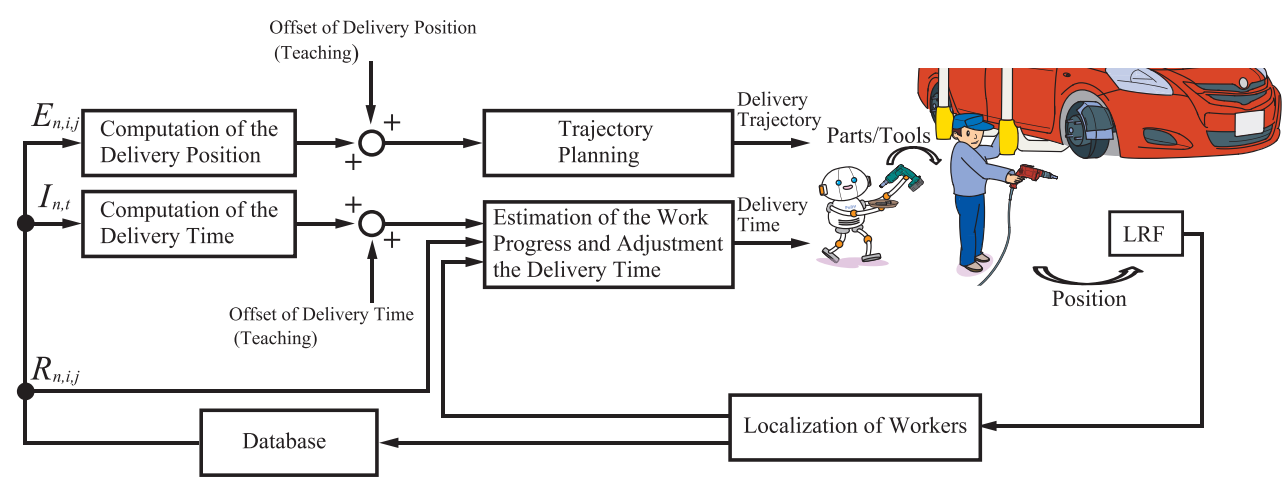

Figure 9

Control System

In the production line, an assembly process is defined by the working process schedule a priori. The working process schedule specifies when each task starts, which part(s) and tool(s) are required for each task, how long it takes to finish each task, and where each task is performed. The worker executes the assembly process according to the working process schedule.

This is mandatory in most of today's automobile production systems and the worker is not allowed to modify the schedule. Thus, it is possible to estimate which task is being performed at each time based on the position of the worker using the execution probability of the $n$-th task for a given cell $(i, j), R_{n, i, j}$.

Based on $R_{n, i, j}$, we can determine which task is most probably performed within each cell $(i, j)$ [3]. That is, each cell is related to a task, which is most probably executed in that cell. Thus, the task most probably executed by the worker can be estimated from the cell, where the worker is located at each time.

Therefore, we can adjust the start of each task as and when necessary (Fig. 8). Note that only when the $n$-th task is completed earlier than estimated using the execution probability for the $n$-th task at time $t, I_{n, t}$, the start time of the $(n+1)$-th task is adjusted by making the start of the task earlier based on the difference observed in real time. The control system, which includes the adjustment of the start of each task, is shown in Fig. 9.

\subsubsection{Experiments}

The control system shown in Fig. 9 was implemented in PaDY P1, and experiments were performed in an actual vehicle assembly line. The robot is controlled by a PD controller. The detailed experimental results are shown in Ref. [3]. When PaDY supported the worker, the time walking to the storage and the length of the worker's path during the task have been decreased. As a result, the time required for the assembly process, which includes six assembly tasks, was reduced by $>25 \%$, by introducing $\mathrm{PaDY}$. 


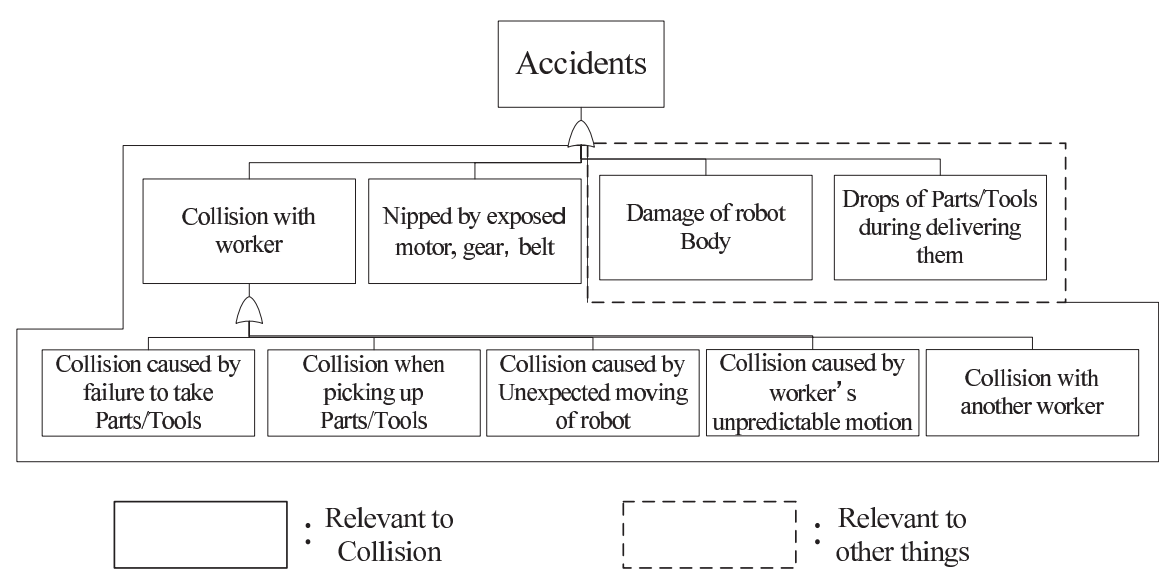

Figure 10

Fault Tree Analysis

\section{Collision Risk Reduction System}

PaDY autonomously operates based on the information from the assembly line. It is installed in the assembly process of the line, and it determines its behavior based on the information from the assembly line, such as when the next vehicle is coming, which types of vehicles are coming, and the worker's location detected by the LRF system.

Even if $\mathrm{PaDY}$ is operating without any problem, the worker engaged in the assembly process may collide with it by being in close proximity with it. The worker may not always be able to see PaDY because they are usually focused on completing the tasks within the working process schedule.

\subsection{Fault Tree Analysis}

Possible hazards leading to an accident in this system were analyzed using fault tree analysis (FTA). The results are shown in Fig. 10. Hazards surrounded by solid lines in Fig. 10 are related to collisions between the worker and the robot, which is the main type of hazard.

It is therefore important to implement measures to prevent collisions between $\mathrm{PaDY}$ and the worker, in order to reduce risks and to prevent nips by exposed mechanisms including motors, gears and belts.

\subsection{Obstacle Detection System}

We designed an obstacle detection system for PaDY. The system comprises multiple proximity sensors distributed on the surface of PaDY's manipulator arm (Figs. 11 and 12). The braking distance required for completely stopping PaDY depends on the attitude and speed of PaDY's manipulator at any given time. For this reason, a 


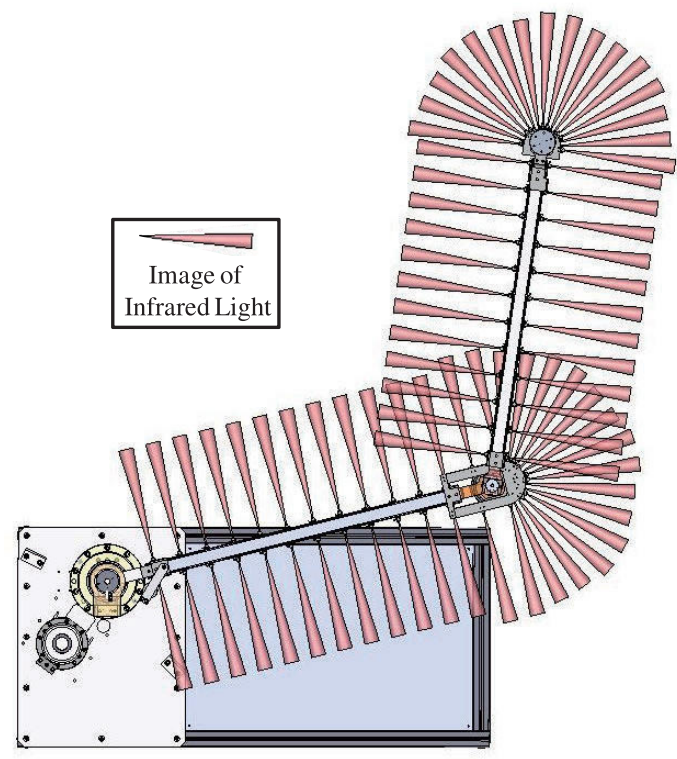

Figure 11

PSD Sensor Arrays on PaDY

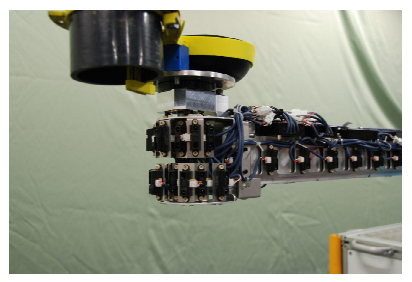

(a)

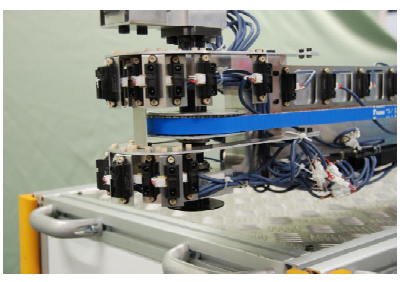

(b)

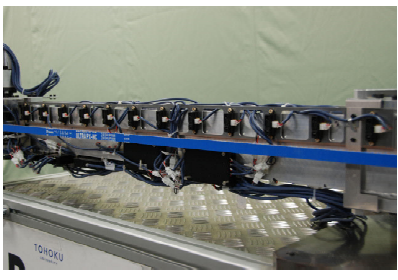

(c)

Figure 12

Parallel Distributed Obstacle Detection System: (a) around End Effector, (b) around Joint, (c) around Links

proximity sensor with variable detection distance (GP2Y0A21YK0F, SHARP) was selected for the obstacle detection sensor.

\subsection{Collision Avoidance System and Reduction of Collision Risks}

The collision avoidance system performs braking control to immediately stop PaDY's manipulator when the obstacle detection system detects an obstacle. Note that each joint is driven by a motor through a mechanical torque limiter in this system.

If the manipulator suddenly stops, the mechanical torque limiters are activated by the inertial force of the manipulator, and the manipulator would be out of control. The braking control need to make the manipulator stop at the shortest possible distance without activating the torque limiters [5].

Even with the collision avoidance system, it is impossible to completely prevent 


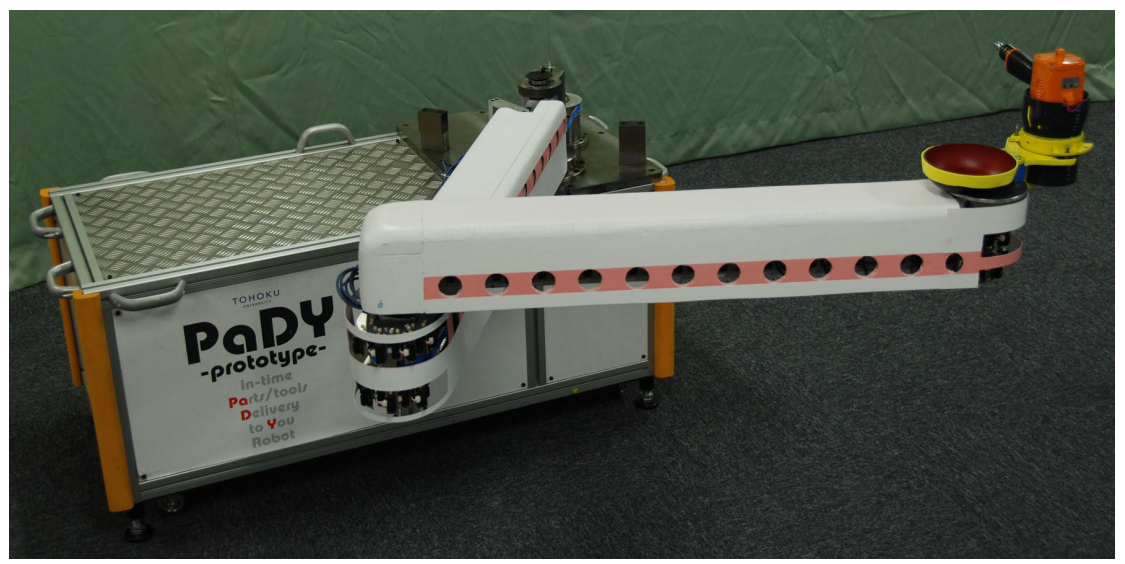

Figure 13

PaDY with Collision Risk Reduction System

collisions. When a collision occurs, it is necessary to reduce the impact and pressing forces after the collision. How much impact force is given to a human from a robot when they collide with each other depends on the magnitude of the moment of inertia. The peak of the impact force becomes large, if the manipulator arm is not covered with a buffer material. K. Suita et al., proposed a concept and a design method of covering a robot with a viscoelastic material to reduce impact force keeping within the human pain tolerance limit[9]. We adopted a similar method proposed by them.

We developed a lightweight arm for PaDY to reduce the moment of inertia and decrease the impact force and attached a buffer material on the surface of PaDY to reduce the peak of the impact force. Fig.13 shows the appearance of the robot arm with the buffer materials on the surface of PaDY. Furthermore, the mechanical torque limiters were installed between the motor outputs and joints of PaDY's manipulator to reduce the pressing force.

PaDY was designed safely to mitigate the possible causes of risks and to meet the safety standards of the company based on Japanese Industrial Standards (JIS B 8433), where PaDY P1 is installed for the further development of PaDY.

\section{Conclusions}

In this article, we have introduced an overview of the Co-Worker Robot called "PaDY", which has been installed in an automotive production system to improve efficiency, reducing the time required to complete the assembly process by $>25 \%$. Different types of PaDYs are under development and will be installed in other assembly processes within the assembly line. We expect that the co-worker robots will be able to assist workers and reduce their working load and thereby increase their overall productivity.

\section{References}

[1] Y. Yamada, H. Konosu, T. Morizono and Y. Umetani: Proposal of SkillAssist: A System of Assisting Human Workers by Reflecting Their Skills in 
Positioning Tasks, Proceedings IEEE International Conference on Systems, Man, and Cybernetics, IV, 1999, pp. 11-16

[2] Naoyuki Takesue, Hideyuki Murayama, Kousyun Fujiwara, Kuniyasu Matsumoto, Hitoshi Konosu, and Hideo Fujimoto: Kinesthetic Assistance for Improving Task Performance -The Case of Window Installation Assist-, International Journal of Automation Technology, Vol.3, No.6, 2009, pp.663670

[3] Jun Kinugawa, Yuta Kawaai, Yusuke Sugahara, and Kazuhiro Kosuge: PaDY : Human-Friendly/Cooperative Working Support Robot for Production Site, Proceedings of the 2010 IEEE/RSJ International Conference on Inteligent Robots and Systems (IROS2010), Taipei 2010, pp. 5472-5479

[4] Jun Kinugawa, Yasufumi Tanaka, Yuta Kawaai, Yusuke Sugahara, and Kazuhiro Kosuge: A Path Generation Method for Collision Risk Reduction and Quantitative Evaluation of Assembly Task Partner Robot, Proceedings of the 2011 IEEE/ASME International Conference on Advanced Intelligent Mechatronics (AIM2011), Budapest 2011, pp. 409-415

[5] Jun Kinugawa, Yuta Kawaai, Yasufumi Tanaka, Yusuke Sugahara, and Kazuhiro Kosuge: Collision Risk Reduction System for Assembly Task Partner Robot, Proceedings of the 2012 IEEE/ASME International Conference on Advanced Intelligent Mechatronics (AIM2012), Kaohsiung 2012, pp. 280285

[6] Yasufumi Tanaka, Jun Kinugawa, and Kazuhiro Kosuge: Motion Planning with Worker's Trajectory Prediction for Assembly Task Partner Robot, Proceedings of the 2012 IEEE/RSJ International Conference on Intelligent Robots and Systems (IROS2012), Vilamoura 2012, pp. 1525-1532

[7] Takahiro Takeda, Yasuhisa Hirata, and Kazuhiro Kosuge: DanceStep Estimation Method Based on HMM for Dance Partner Robot, IEEE Transaction Industrial Electronics, vol. 54, no. 2, 2007, pp.699-706

[8] Philip J. Clark and Francis C. Evans: Distance to Nearest Neighbor as a Measure of Spatial Relationships in Populations, Ecology, Vol.35, No.4, 1954, pp.445-453

[9] K. Suita, Y. Yamada, N. Tuchida, K. Imai, H. lkeda, N. Sugimoto: Failureto-Safety "Kyozon" System with Simple Contact Detection and Stop Capabilities for Safe Human-Autonomous Robot Coexistence, Proc. IEEE International Conference on Robotics and Automation, 1995, pp. 3089-3096 